\title{
Un nuovo farmaco per l'iperfosfatemia
}

\author{
Sandro Mazzaferro, Silverio Rotondi, Lida Tartaglione, Maria Luisa Muci, Marzia Pasquali
}

Dipartimento di Scienze Cardiovascolari Respiratorie Nefrologiche Anestesiologiche e Geriatriche, Università degli Studi di Roma "La Sapienza", Roma

\begin{abstract}
A new hyperphosphatemia drug
Chronic renal failure has an average prevalence of $10 \%$ in the general population and carries a significant burden of morbidity and mortality. Moreover, the recently appreciated cross-talk between bone and the kidney is considered to be involved, when deranged, in the process of ectopic and vascular calcification. By disrupting this cross-talk, renal insufficiency can be responsible for cardiovascular disease. Vascular calcifications, which are increased in renal insufficiency, are also considered a major cause of cardiovascular disease. Hyperphosphatemia or an excessive phosphate burden in general is regarded as a formidable pathogenetic factor. We have known since many years that, by regulating tubular filtration and excretion, renal function plays a principal role in phosphate homeostasis. Also, in more recent years we have learned that the kidney synthesizes Klotho, a coreceptor of FGF23 which, in turn, is a major phosphate-regulating hormone produced by osteocytes. With these premises, hyperphosphatemia has taken center stage in renal patients and the attention to treatment of hyperphosphatemia has increased. In fact, the number of available oral phosphate binders has grown and a new class of iron-based drugs is emerging. Given the metal nature of iron and its positive charge, it is not surprising that iron is a powerful phosphate binder. Two such drugs have been approved for use in renal patients: sucroferric oxyhydroxide (or PA21) and iron citrate. Since only the former is available in Italy, we limited the present review to the analysis of the available clinical data useful to recognize the strengths and limitations of this new drug.
\end{abstract}

Keywords: Chronic Kidney Disease, Chronic Renal Failure, Hyperphosphatemia, Mineral Bone Disorders, Phosphate Binders, Sucroferric-Oxy-Hydroxide

La malattia renale cronica ha assunto rilevanza sanitaria globale da quando sono state evidenziate la sua ampia diffusione (circa il $10 \%$ della popolazione generale) e la sua stretta associazione con un'aumentata mortalità e morbilità. In particolare è emerso il legame funzionale che il rene ha con altri parenchimi, tra i quali certamente speciale risulta quello con l'osso (1). Il legame tra rene e osso è importante perché responsabile della regolazione dei livelli di fosforemia. Infatti, il rene è il principale escretore dei fosfati in eccesso assorbiti con la dieta, ed è anche il principale produttore di Klotho, una proteina dalle azioni molteplici e in parte da riconoscere, il cui ruolo fisiologico più accreditato è di funzionare

Accepted: July 18, 2016

Published online: August 31, 2016

Indirizzo per la corrispondenza:

Dr. Sandro Mazzaferro

Dipartimento di Scienze Cardiovascolari Respiratorie Nefrologiche

Anestesiologiche e Geriatriche,

Università degli Studi di Roma "La Sapienza"

V.le del Policlinico, 155

00185 Roma

sandro.mazzaferro@uniroma1.it da co-recettore dell'ormone oggi ritenuto principale regolatore della escrezione di fosfati: I'FGF23 (o Fibroblast Growth Factor 23). È importante sottolineare che l'FGF23 è prodotto principalmente dagli osteociti e che è anche responsabile della sintesi di vitamina $D$ attiva che, a sua volta, dei fosfati regola l'assorbimento intestinale. Nel corso della insufficienza renale i livelli di Klotho si riducono assai precocemente (2), e si associano ad aumento di FGF23 probabilmente per superare la resistenza renale all'FGF23. L'attenzione che oggi si dà ai livelli di fosforemia è secondaria alla evidenza della stretta associazione dei livelli circolanti di fosforo con le calcificazioni vascolari e la mortalità da tutte le cause e cardiovascolare, ma anche con la evolutività della malattia renale (3). Per questo motivo il fosforo è stato proposto come terzo componente, dopo proteinuria e ipertensione, dei fattori di progressione della insufficienza renale (4). Non deve dunque meravigliare che l'attenzione terapeutica alla iperfosforemia nel nefropatico sia di molto aumentata negli ultimi anni. Una testimonianza dell'accresciuto interesse si intuisce dall'incremento dei farmaci chelanti del fosforo dietetico disponibili in commercio. I chelanti del fosforo, insieme alle indicazioni dietetiche e alla efficienza dialitica, rappresentano infatti uno dei tre cardini della terapia della iperfosforemia nei soggetti con insufficienza renale terminale. Fino a non molti anni fa il 
TABELLA I-Tipi di chelanti del fosforo di possibile impiego in nefrologia

Contenenti alluminio

A base di calcio

Sevelamer

Carbonato di lantanio

Sali di magnesio

Carbonato di magnesio e ferro

Acido nicotinico

Chitosano

Colestilan

Citrato ferrico

Ossi-idrossido Sucroferrico

Inibitori della pompa $\mathrm{NaPi} 2$

nefrologo aveva a disposizione solamente un paio di chelanti del fosforo: a base di alluminio o a base di calcio. Negli ultimi anni, come illustrato nella Tabella I, la lista si è sensibilmente allungata ed è destinata a crescere, rendendo necessaria la scelta tra circa una dozzina di prodotti.

La novità più recente tra i chelanti del fosforo è certamente rappresentata dai prodotti a base di ferro (Fe). II ferro è l'elemento numero 26 della Tavola periodica ed è anche il metallo più abbondante della terra. È presente in varie forme ossidate, le più comuni sono il ferroso $(\mathrm{Fe} 2+)$ e il ferrico $(\mathrm{Fe} 3+)$. Il ferro è essenziale per la vita di tutti gli esseri viventi poiché svolge numerose funzioni biologiche e la sua carenza è causa di malattia. Tuttavia, come per altri metalli, anche un suo eccesso può essere tossico, come dimostrato dalle patologie di accumulo nell'uomo. Per essere assorbito dagli alimenti a livello intestinale, deve essere nella forma ferrosa. Essendo un elemento a carica positiva, non deve sorprendere la sua capacità legante per il fosfato ( $\left.\mathrm{PO}_{4} 2-\right)$ che ha invece carica negativa.

I chelanti del fosforo a base di Fe sono stati in fase di studio per molti anni e di recente due prodotti hanno ricevuto l'approvazione da parte della FDA (Food and Drug Administration): l'ossi-idrossido sucroferrico (noto anche come PA21) e il Fe citrato. Nella presente revisione ci occuperemo solo del primo poiché è l'unico disponibile per la prescrizione in Italia.

L'ossi-idrossido sucroferrico è composto da una miscela di ferro ferrico, ossigeno e residui idrossili (da qui il nome ossiidrossi) che assume una forma polinucleare grazie ai legami a ponte formati dagli idrossili (Fig. 1). L'aggiunta di saccarosio e amidi serve a formare una sorta di guscio che stabilizza la molecola, impedisce la degradazione del ferro e ne preserva la capacità chelante del fosforo. Nel farmaco PA21 troviamo le seguenti proporzioni molari: ferro: $21 \%$, saccarosio: $30 \%$, amidi: $28 \%$, acqua: $10 \%$ (Fig. 1). Prove in vitro hanno evidenziato che l'ossi-idrossido sucroferrico ha una elevata affinità e capacità legante per il fosfato nel range di pH presenti a livello intestinale (5). L'elevata affinità limita il rilascio del ferro che è praticamente assente a stomaco pieno ed evidenziabile solo in minima quantità in condizioni di stomaco vuoto (5). Occorre comunque ricordare che il Fe liberato è nella forma ferrica e quindi poco assorbibile. In ogni caso, dato che si tratta di un farmaco con alte concentrazioni dell'elemento e del quale è prevedibile una somministrazione cronica per lunghi periodi, il primo studio di sicurezza richiesto dalle autorità è stato relativo alla valutazione dell'entità di assorbimento del Fe. L'uptake marziale è stato valutato in uno studio di fase I

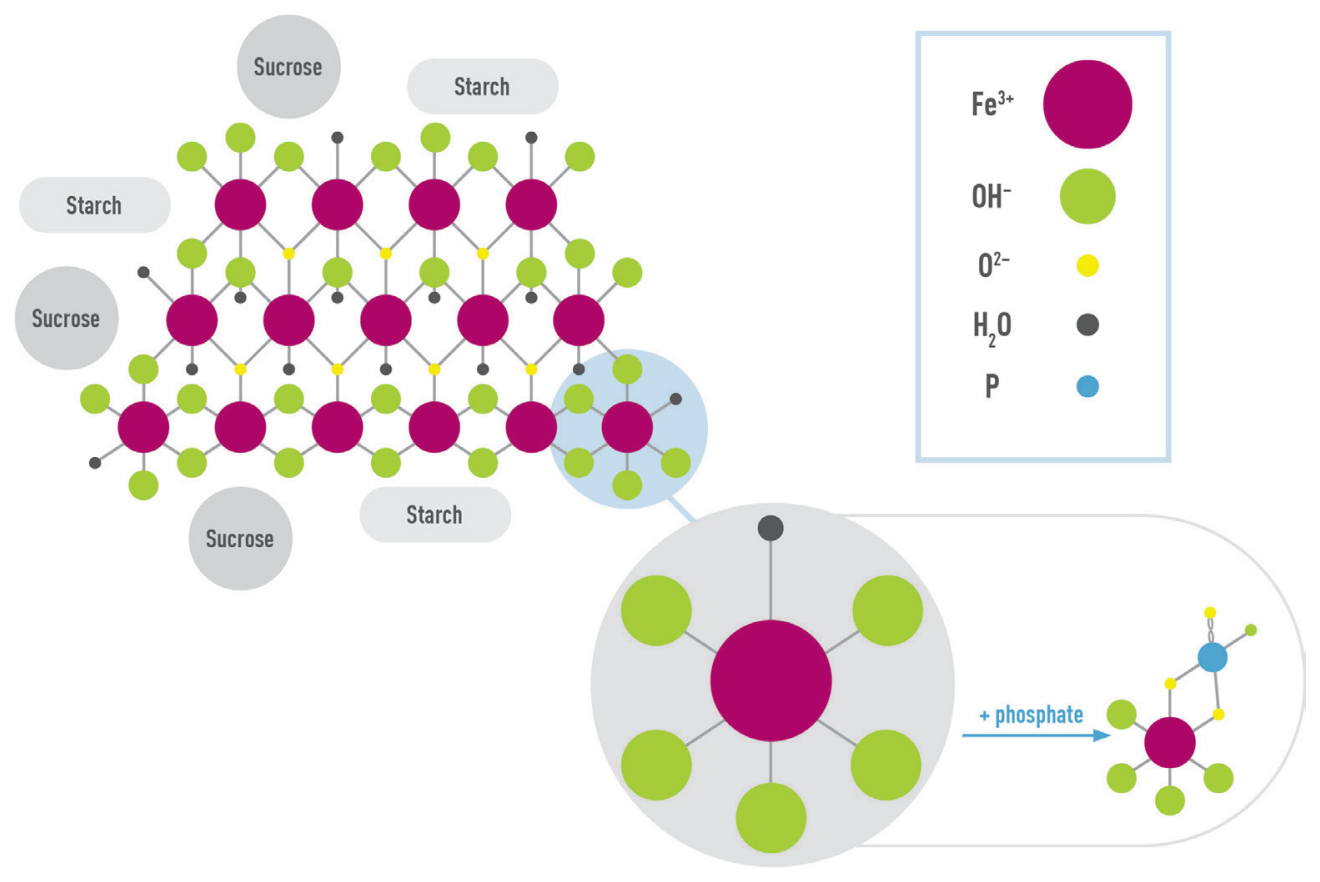

Fig. 1 - II sucroferri-ossiidrossido è una molecola composta che contiene una miscela di Ferro, Idrossili ed acqua ed è stabilizzata con saccarosio ed amido. Mostra elevata affinità legante per il fosfato (che va a sostituire un idrossile nella molecola) in un ampio range di $\mathrm{pH}$ ed un assai limitato release del ferro legato. È stato calcolato che $500 \mathrm{mg}$ legano circa $130 \mathrm{mg}$ di fosfato. Starch $=$ amido, Sucrose $=$ Saccarosio, Phosphate $=$ fosfato 
(6). Il farmaco è stato somministrato alla dose di $10 \mathrm{~g} /$ die per 7 giorni a 8 pazienti con IRC stadio 3-4, a 8 pazienti in emodialisi e a 8 soggetti normali. Una singola dose del farmaco era radiomarcata con ${ }^{59} \mathrm{Fe}$. L'assorbimento di ferro misurato con questo metodo è stato dello 0,06\% nella IRC (Insufficienza Renale Cronica), dello $0,02 \%$ in emodialisi e dello $0,43 \%$ nei normali, senza nessun cambiamento dei livelli sierici di sideremia, transferrina e ferritina. Al termine della somministrazione si è invece registrata una riduzione significativa dei livelli plasmatici di fosfato nei pazienti renali ma non nei soggetti normali. In altri termini, l'assorbimento di Fe dal PA21 è trascurabile e risulta persino ridotto in presenza di insufficienza renale cronica, mentre se ne conferma la capacità chelante del fosfato.

Lo studio successivo, di fase II, ha avuto come obiettivo primario la ricerca della dose adeguata e come obiettivi secondari l'efficacia, la sicurezza e la tollerabilità (7). Si è trattato di uno studio multicentrico che ha coinvolto 50 Centri europei e americani con approccio randomizzato, open-label. I pazienti arruolati erano in emodialisi e hanno ricevuto il farmaco a dosi fisse nei vari gruppi considerati, ma crescenti e pari a 0,25-1,0-1,5-2,0 o 2,5 g al giorno. II gruppo di controllo riceveva la terapia chelante con il farmaco competitor, costituito in questo caso dal Sevelamer- $\mathrm{HCl}$, prescritto anch'esso a dose fissa di 4,8 $\mathrm{g}$ al giorno. L'obiettivo primario era rappresentato dalla modifica dei livelli di fosforo. Dopo la fase di screening, i pazienti selezionati hanno sospeso per 2 settimane la loro terapia chelante precedente e sono stati quindi randomizzati nello studio solo se superavano il valore di 5,5 $\mathrm{mg} / \mathrm{dL}$ di fosforemia. La randomizzazione ha interessato 154 pazienti, che sono stati divisi in 5 gruppi diversi, composti ciascuno da almeno 25 pazienti. Le dosi fisse crescenti di chelante sono state somministrate per un periodo di 6 settimane. Il sesto gruppo era quello di controllo, trattato con il Sevelamer. Nel corso dello studio si sono registrati 51 drop-out dal trattamento per vari motivi inclusa l'ipofosforemia in 10 casi. Tutti i dosaggi di ossi-idrossido sucroferrico impiegati, con la sola eccezione della dose di 0,25 g/die, hanno prodotto una significativa riduzione dei livelli di fosforemia. Si è osservata una tendenza all'aumento della efficacia con l'aumentare delle dosi, con effetto massimo alle due dosi più elevate. In particolare le dosi di 1,0 e 1,5 g/die hanno prodotto una riduzione della fosforemia simile a quella ottenuta nel gruppo Sevelamer, suggerendo una corrispondenza delle rispettive dosi. II calo della fosforemia è risultato significativo già dopo una settimana dall'inizio del trattamento, risultato peraltro non diverso statisticamente da quanto osservato con Sevelamer, con il quale il calo è stato registrato dopo 2 settimane. Altro risultato significativo in questo studio è la conferma dell'assenza di effetti sul patrimonio marziale. Infatti, i livelli di sideremia e ferritina e la saturazione della transferrina non hanno mostrato cambiamenti significativi rispetto al basale in nessuno dei gruppi terapeutici, analogamente a quanto osservato nel gruppo di controllo. Anche la percentuale di pazienti che ha avuto effetti collaterali è risultata non diversa tra i trattati $(60,7 \%)$ e i controlli $(59,7 \%)$. Curiosamente, un evento avverso frequente con l'ossi-idrossido sucroferrico è stata l'ipofosforemia, che in alcuni casi ha rappresentato, in ottemperanza al protocollo, motivo di sospensione del trattamento. Come con altre terapie orali a base di chelanti, gli eventi avversi gastrointestinali sono risultati frequenti (pari al 22,7\% nei trattati e al $26,9 \%$ nei controlli) ma mediamente inferiori ad altri studi. In ogni caso, questo studio ha dimostrato che è possibile ottenere il controllo della fosforemia in una percentuale elevata di pazienti in emodialisi con dosi superiori ai $1,0 \mathrm{~g} /$ die già dopo una settimana di trattamento e con eventi avversi non superiori a quanto si osserva con altri chelanti.

Se lo studio di fase II ha individuato la dose efficace e la non inferiorità rispetto a un competitor, lo studio successivo di fase III ha avuto il compito di dimostrare l'efficacia e la sicurezza del nuovo farmaco su un numero consistente di pazienti (8). Il protocollo prevedeva come obiettivi principali la dimostrazione della superiorità della dose di mantenimento, rispetto alla dose minima, nel controllare la fosforemia e la conferma della non inferiorità rispetto al competitor Sevelamer. Lo studio è stato condotto in 174 Centri degli Stati Uniti, d'Europa e di Russia, Ucraina e Sud Africa e ha arruolato oltre 1000 pazienti sia in emodialisi che in dialisi peritoneale. Dopo lo screening, era previsto un periodo di wash-out dalla terapia chelante precedente, della durata di 4 settimane. I casi che in questa fase sviluppavano iperfosforemia venivano randomizzati nel rapporto di 2:1 a Sucroferri-ossi-idrossido (710 pazienti) oppure a Sevelamer carbonato (349 pazienti). Seguiva una fase di titolazione della dose, che per il chelante marziale era compreso tra 1 e $3 \mathrm{~g} /$ die e per il Sevelamer tra 4,8 e $14,4 \mathrm{~g} /$ die, partendo per entrambi dalla dose minore. Dopo la fase di titolazione, della durata di 4 settimane, seguivano 20 settimane di mantenimento della dose efficace con verifica dei livelli fosforemici raggiunti. A questo punto era prevista una seconda fase dello studio durante la quale un sottogruppo del trattamento attivo è stato ulteriormente suddiviso in due gruppi e per un periodo di 3 settimane ha continuato la dose di mantenimento di PA21 oppure una dose ridotta pari a $0,250 \mathrm{~g} /$ die (cioè la dose risultata inefficace nello studio di fase II). Durante la fase iniziale di titolazione del farmaco, nella quale l'obiettivo era il raggiungimento di una fosforemia $<5,5 \mathrm{mg} / \mathrm{dL}$, la dose media prescritta di farmaci è stata pari a circa 1,4 g/die di PA21 e circa 6,2 g/die per Sevelamer. La riduzione della fosforemia alla $12^{\text {a }}$ settimana era pari a $-2,1$ $\mathrm{mg} / \mathrm{dL}$ e a $-2,4 \mathrm{mg} / \mathrm{dL}$ rispettivamente con Sucroferri-ossiidrossido e con Sevelamer, consentendo di affermare la non inferiorità del primo farmaco rispetto al secondo. Al termine della prima fase (mantenimento del dosaggio, $24^{\mathrm{a}}$ settimana), sono stati individuati i due gruppi per il proseguimento nella seconda fase, a dosaggi diversi, dello studio. I due gruppi scelti avevano raggiunto livelli paragonabili di fosforemia, e hanno continuato l'osservazione per ulteriori 3 settimane con la dose di mantenimento (50 pazienti) o con la dose ridotta (49 pazienti) pari a 0,250 g/die. Mentre nel primo gruppo la 
fosforemia non si è modificata, nel secondo si è registrato un incremento medio significativo pari a circa $1,8 \mathrm{mg} / \mathrm{dL}$. Questo studio, tra i più importanti in nefrologia per numero di pazienti coinvolti, ha consentito di fare anche altre osservazioni clinicamente rilevanti. Anzitutto, una importante differenza pratica: il numero di compresse prescritte è risultato nettamente più basso con il PA21 (3,1 compresse al dì rispetto a 8,1 del competitor). Quanto agli effetti collaterali insorti in rapporto alla terapia, la percentuale di pazienti che ha avuto almeno un evento è risultata maggiore nel gruppo di trattamento $(83,2 \%)$ rispetto al controllo $(76,1 \%)$. In particolare nel gruppo PA21 sono risultati più frequenti alcuni eventi (diarrea: $20,1 \%$ vs $7,5 \%$; feci colorate: $15,4 \%$ vs $0,3 \%$; iperfosforemia: $11,2 \%$ vs $7,8 \%$ ) mentre nel gruppo controllo hanno prevalso altri eventi (stipsi: $3,8 \%$ vs $7,2 \%$; nausea: $7,2 \%$ vs $11,2 \%$ ). In ogni caso gli eventi avversi severi o gravi riferiti al trattamento sono risultati limitati e pari all'1,3\% con PA21 e all'1,1\% con Sevelamer, senza differenze significative. Gli eventi avversi gastrointestinali sono stati la causa principale di interruzione della terapia, in proporzione maggiore nel gruppo attivo $(54,0 \%$ vs $43,5 \%)$. In proposito è interessante notare che l'evento più frequente, la diarrea (peraltro intesa come feci molli) ha avuto la massima prevalenza nella fase di titolazione della dose del farmaco e ha comportato la sospensione del trattamento in casi limitati $(2,8 \%$ vs $0,6 \%)$. I parametri marziali e i livelli di emoglobina non hanno mostrato cambiamenti durante il follow-up, né differenze significative tra il gruppo attivo e quello di controllo, con la sola eccezione della transferrina risultata più elevata alla $24^{\mathrm{a}}$ settimana nel gruppo PA21. Tuttavia I'uso del ferro endovena non era limitato in nessuno dei gruppi durante lo studio.

Per questo studio di fase III era prevista una estensione per portare a 52 settimane il follow-up totale di 391 pazienti nel braccio attivo e 268 nel braccio di controllo (9). I dati demografici di questi due gruppi all'inizio della fase di estensione erano sovrapponibili. I livelli di fosforemia sono rimasti stabili per tutto il periodo di osservazione senza differenze tra i due trattamenti e hanno consentito di mantenere livelli compresi nel range raccomandato dalle linee guida KDOQI. Anche durante questa fase di estensione il controllo della fosforemia è stato raggiunto con un numero di compresse significativamente inferiore nel gruppo PA21 (in media 4 comprese) rispetto al gruppo Sevelamer (in media 10 compresse). Durante lo studio in entrambi i gruppi terapeutici si è registrato un aumento significativo dei livelli di PTH (da $40,0 \pm 30$ a $46,1 \pm 40 \mathrm{pmol} / \mathrm{L}$ nel gruppo PA21 e da $39.3 \pm 28,4$ a $46,0 \pm 34,9 \mathrm{pmol} / \mathrm{L}$ nel gruppo Sevelamer) a fronte di una minima riduzione dei livelli di fosfatasi alcalina ossea (da $18,6 \pm 15,4$ a $16,5 \pm 14,4 \mathrm{ng} / \mathrm{mL}$ nel gruppo PA21 e da $20,0 \pm 17,2$ a $17,7 \pm 14,5 \mathrm{ng} / \mathrm{mL}$ nel gruppo Sevelamer) e di una sostanziale stabilità dei livelli calcemici. I dati di followup del lungo periodo hanno confermato la non influenza di entrambe le terapie chelanti sui parametri biochimici del metabolismo marziale. Gli eventi avversi registrati nel lungo periodo non sono risultati differenti tra i due bracci terapeutici.

Un altro studio disponibile, utile a esaminare le caratteristiche cliniche del PA21, è quello che ha valutato l'interferenza nell'assorbimento di altri farmaci (10). Sono stati misurati la concentrazione massima e l'AUC (Area Under the Curve) di losartan, furosemide, digossina, omeprazolo e warfarin da soli e in contemporanea all'assunzione di PA21 ed è stata dimostrata la non interferenza nell'assorbimento.

Pertanto, sulla base degli studi finora disponibili, è possibile considerare il nuovo chelante a base di ferro come non inferiore al competitor Sevelamer, con un profilo di sicurezza e tollerabilità sovrapponibile, e con il vantaggio di un numero minore di compresse da assumere.

\section{Disclosures}

Financial support: This Supplement has been supported by an unrestricted educational grant provided by Vifor Fresenius Medical Care Renal Pharma Italia S.r.l.

Conflict of interest: The authors declare they do not have any conflict of interest.

\section{Bibliografia}

1. Mazzaferro S, Pasquali M, Pirrò G, Rotondi S, Tartaglione L. The bone and the kidney. Arch Biochem Biophys 2010;503(1):95-102.

2. Rotondi S, Pasquali M, Tartaglione L, Muci ML, Mandanici G, Leonangeli C, Sales S, Farcomeni A, Mazzaferro S. Soluble a-Klotho Serum Levels in Chronic Kidney Disease. Int J Endocrinol 2015;2015:872193.

3. Zoccali C, Ruggenenti P, Perna A, Leonardis D, Tripepi R, Tripepi G, Mallamaci F, Remuzzi G; REIN Study Group. Phosphate may promote CKD progression and attenuate renoprotective effect of ACE inhibition. J Am Soc Nephrol 2011;22(10):1923-30.

4. Cozzolino M, Gentile G, Mazzaferro S, Brancaccio D, Ruggenenti P, Remuzzi G. Blood pressure, proteinuria, and phosphate as risk factors for progressive kidney disease: a hypothesis. Am J Kidney Dis 2013;62(5):984-92.

5. Wilhelm M, Gaillard S, Rakov V, Funk F. The iron-based phosphate binder PA21 has potent phosphate binding capacity and minimal iron release across a physiological $\mathrm{pH}$ range in vitro. Clin Nephrol 2014; 81(4): 251-8.

6. Geisser P, Philipp E. PA21: a novel phosphate binder for the treatment of hyperphosphatemia in chronic kidney disease. Clinical Nephrology 2010;74(1): 4-11.

7. Wüthrich RP, Chonchol M, Covic A, Gaillard S, Chong E, Tumlin JA. Randomized Clinical Trial of the Iron-Based Phosphate Binder PA21 in Hemodialysis Patients. Clin J Am Soc Nephrol 2013;8: 280-9.

8. Floege J, Covic AC, Ketteler M, Rastogi A, Chong EM, Gaillard S, Lisk LJ, Sprague SM; PA21 Study Group. A phase III study of the efficacy and safety of a novel iron-based phosphate binder in dialysis patients. Kidney Int. 2014;86(3):638-47.

9. Floege J, Covic AC, Ketteler M, Mann JF, Rastogi A, Spinowitz B, Chong EM, Gaillard S, Lisk LJ, Sprague SM; Sucroferric Oxyhydroxide Study Group. Long-term effects of the iron-based phosphate binder, sucroferric oxyhydroxide, in dialysis patients. Nephrol Dial Transplant 2015;30(6):1037-46.

10. Chong E, Kalia V, Willsie S, Winkle P. Drug-drug interactions between sucroferric oxyhydroxide and losartan, furosemide, omeprazole, digoxin and warfarin in healthy subjects. J Nephrol 2014;27(6):659-66. 